\title{
El paciente geriátrico en urgencias
}

\section{The geriatric patient and emergency care}

\author{
F.J. Martín-Sánchez ${ }^{1}$, C. Fernández Alonso', C. Merino²
}

\section{RESUMEN}

La demanda en la atención urgente ha ido creciendo progresivamente en los últimos años y este incremento es más acusado en la población anciana. Teniendo en consideración que el paciente anciano requiere evaluaciones más complejas con una mayor realización de pruebas complementarias y consultas a otros especialistas, estancias más prolongadas en urgencias y mayor porcentaje de ingresos, el progresivo envejecimiento poblacional puede llegar a repercutir seriamente en la presión asistencial de los servicios de urgencias hospitalarios (SUH).

El paciente anciano de alto riesgo es vital detectarlo antes de asignarle una ubicación definitiva. Para ello, es importante instaurar un cribado entre los pacientes ancianos que acuden a urgencias con el fin de seleccionar a los que se van a beneficiar de una valoración geriátrica integral y así poder diseñar un plan específico de cuidados. La intervención urgente en el paciente geriátrico no debe afrontarse exclusivamente como un problema médico sino que debe tenerse en cuenta aspectos funcionales, mentales o sociales. Representa un reto para los SUH.

En el presente artículo vamos a desarrollar diversos aspectos como la detección y la valoración del paciente geriátrico así como a establecer ciertas recomendaciones para el entorno de urgencias.

Palabras clave. Emergencias. Geriatría. Fragilidad. Anciano. Valoración.

\begin{abstract}
Demand in emergency care has been growing progressively in recent years and this increase is more pronounced in the elderly population. Taking into account that the elderly patient requires more complex evaluations with a greater requirement for complementary tests and consultations with other specialists, longer stays in the emergency ward and a greater percentage of admissions, the progressive ageing of the population might come to have a serious repercussion on hospital emergency departments.

It is vital to detect high risk elderly patients before assigning them a definitive placement. For this purpose it is important to install a sieving process amongst elderly patients who attend the emergency department in order to select those that will benefit from a comprehensive geriatric assessment and thus be able to design a specific care plan. Emergency intervention in elderly patients should not be faced exclusively as a medical problem, but functional, mental or social aspects should be taken into account. This represents a challenge for emergency care.

This article considers different aspects such as the detection and assessment of the geriatric patient, as well as establishing certain recommendations for emergency setting.
\end{abstract}

Key words. Emergency care. Geriatric. Frailty. Elderly. Assessment.
1. Servicio de Urgencias. Hospital Clínica San Carlos. Madrid.

2. Servicio de Urgencias. Hospital Virgen del Camino. Pamplona.

\author{
Correspondencia \\ Francisco Javier Martín-Sánchez \\ Servicio de Urgencias \\ Hospital Clínico San Carlos \\ Profesor Martín-Lagos, s/n \\ 28040 Madrid \\ E-mail: fjjms@hotmail.com
}




\section{INTRODUCCIÓN}

El envejecimiento poblacional ha tenido un impacto significativo en la salud pública y en la atención sanitaria. En los países desarrollados el sector de la población anciana es el que ha sufrido una mayor tasa de crecimiento en las últimas décadas. En España representa actualmente el $20 \%$ y se estima que para el año 2050 alcance el 30\% de la población general. Este incremento porcentual será aún mayor en el grupo de los mayores de 85 años.

Los servicios de urgencias hospitalarios (SUH) son la puerta de mayor facilidad de acceso al sistema sanitario público. En los últimos años, como consecuencia del crecimiento progresivo de la demanda en la atención sanitaria urgente, se viven a menudo situaciones de saturación y, en ocasiones, de colapso, que deterioran la efectividad y la calidad asistencial ${ }^{1}$.

Estudios recientemente publicados que analizaron el uso de las urgencias han evidenciado un incremento progresivo del número absoluto de las visitas a urgencias, que es más acusado en la población mayor de 65 años $^{2,3}$. Los datos actualmente disponibles calculan que el paciente anciano representa más del 15-25\% de las consultas urgentes ${ }^{4}$. En el área de salud de Pamplona, el porcentaje de urgencias hospitalarias atendidas en la población anciana durante el año 2008 osciló, en función del centro hospitalario, entre 20 y el $40 \%$ del total (Tabla 1).

Tabla 1. Datos sobre los pacientes ancianos atendidos en el área hospitalaria de Pamplona en el año 2008.

\begin{tabular}{|c|c|c|c|c|c|c|c|c|}
\hline & \multicolumn{3}{|c|}{ Datos de atención global } & \multicolumn{5}{|c|}{ Pacientes?65 años } \\
\hline & Pacientes & Ingresos & Observación & Total & $\begin{array}{c}\text { Visita a } \\
\text { Urgencias } \\
\text { (\%) }\end{array}$ & $\begin{array}{c}\text { No de } \\
\text { ingresos } \\
(\%)\end{array}$ & $\begin{array}{l}\text { Porcentaje } \\
\text { sobre total } \\
\text { de ingresos }\end{array}$ & $\begin{array}{c}\text { № de observaciones } \\
\text { (\% del total de } \\
\text { observación) }\end{array}$ \\
\hline $\begin{array}{l}\text { Hospital } \\
\text { Virgen del } \\
\text { Camino }\end{array}$ & 60.610 & $\begin{array}{c}8.121 \\
13,39 \%\end{array}$ & $\begin{array}{c}2085 \\
3,44 \%\end{array}$ & 14.032 & $23,15 \%$ & $\begin{array}{c}4852 \\
34,57 \%\end{array}$ & $59,74 \%$ & $\begin{array}{c}851 \\
40,81 \%\end{array}$ \\
\hline $\begin{array}{l}\text { Hospital } \\
\text { de } \\
\text { Navarra }\end{array}$ & 55.754 & $\begin{array}{c}9593 \\
17,2 \%\end{array}$ & $\begin{array}{c}3309 \\
5,93 \%\end{array}$ & 21.715 & $38,94 \%$ & $\begin{array}{c}5993 \\
27,60 \%\end{array}$ & $62,47 \%$ & $\begin{array}{c}1659 \\
50,13 \%\end{array}$ \\
\hline $\begin{array}{c}\text { Datos } \\
\text { globales } \\
\text { área de } \\
\text { Pamplona }\end{array}$ & 116.364 & $\begin{array}{c}17714 \\
15,22 \%\end{array}$ & $\begin{array}{c}5394 \\
4,63 \%\end{array}$ & 35.747 & $30,71 \%$ & $\begin{array}{l}10845 \\
30,33 \%\end{array}$ & $61,22 \%$ & $\begin{array}{c}2510 \\
46,53 \%\end{array}$ \\
\hline
\end{tabular}

Considerando que el paciente anciano se asocia a unas estancias más prolongadas en urgencias ${ }^{5}$ y a un mayor porcentaje de ingresos ${ }^{6}$, el incremento progresivo de las visitas a urgencias en dicho sector poblacional puede tener un tremendo impacto en la presión asistencial de los $\mathrm{SUH}^{2,3}$.

Teniendo en cuenta lo anteriormente expuesto, el paciente geriátrico representa hoy día un reto para los SUH. Por ello, en el presente artículo vamos a desarrollar a continuación diversos aspectos como la detección y la valoración del paciente geriátrico así como establecer ciertas recomendaciones en dicho perfil de pacientes en el entorno de urgencias.

\section{EL PACIENTE GERIÁTRICO EN URGENCIAS}

A lo largo de los años, y en función de la fuente consultada, los conceptos de anciano, anciano frágil o paciente geriátrico varían e incluso en algunas ocasiones se han utilizado de forma indistinta. 
Por paciente geriátrico se entiende aquel sujeto de edad avanzada con pluripatología y polifarmacia y que además presente cierto grado de dependencia para las actividades básicas de la vida diaria. Es frecuente que asocie problemas cognitivos o afectivos y la necesidad de recursos sociosanitarios. Por tanto, es evidente que la definición de paciente geriátrico engloba una serie de aspectos médicos, psicológicos, funcionales y sociales de los cuales dista el mero término de anciano.

\begin{abstract}
Algunos autores distinguen el concepto de anciano frágil. El anciano frágil es aquel paciente de edad avanzada que presenta una serie de cambios consecuencia del envejecimiento en los diferentes órganos y sistemas que le otorgan una pérdida de la reserva fisiológica. Dicho paciente conserva la independencia para las actividades básicas de la vida diaria, pero ante la presencia de un proceso intercurrente está en riesgo de sufrir un deterioro de la capacidad funcional $^{7,8}$ (Tabla 2).
\end{abstract}

Tabla 2. Criterios de fragilidad de Fried.

Pérdida de peso no intencionada

5 kilogramos o bien $>5 \%$ del peso corporal en el último año.

\section{Debilidad muscular}

Fuerza prensora $<20 \%$ del límite de la normalidad ajustado por sexo y por índice de masa corporal.

\begin{tabular}{l} 
Baja resistencia-cansancio \\
Autorreferido por la misma persona e identificado por dos preguntas de la escala CES-D. (Center \\
Epidemiological Studies-Depression) \\
\hline Lentitud de la marcha \\
$\quad$ Velocidad de la marcha, para recorrer una distancia de $4,5 \mathrm{~m}<20 \%$ del límite de la normalidad ajustado \\
por sexo y altura. \\
Nivel bajo de actividad física \\
$\quad$ Cálculo del consumo de calorías semanales por debajo del quintil inferior ajustado por sexo.
\end{tabular}

La presencia de 3 ó más de estos criterios nos indica fragilidad.

El anciano suele hacer un uso adecuado de las urgencias. Cuando comparamos las visitas a urgencias en los grupos de edad de mayores o menores de 65 años, se evidencia que el paciente anciano tiene mayor probabilidad de sufrir un problema de gravedad. De hecho, son más frecuentemente clasificados con un nivel de atención de muy urgentes y urgentes ${ }^{9,10}$, y el porcentaje de ingresos es mayor indistintamente del motivo de consulta o el nivel de urgencias ${ }^{9}$.

Los motivos más frecuentes de consulta a urgencias en los ancianos son los síntomas generales y los problemas digestivos, respiratorios y músculo-esqueléticos ${ }^{10}$. En un estudio reciente que incluyó a más 50.000 atenciones urgentes en un hospital terciario español, se documentó que los motivos de atención urgente más frecuentes en la población anciana eran el malestar general, la disnea, el dolor abdominal, los problemas en extremidades, el dolor to- rácico y los problemas urinarios ${ }^{9}$. De hecho las causas que engloban la mayor mortalidad en los ancianos son las enfermedades del corazón, el cáncer, la enfermedad cerebrovascular y las enfermedades crónicas del aparato respiratorio ${ }^{10}$.

Los pacientes de edad avanzada tienen con mayor frecuencia un consumo más elevado de fármacos. Esta circunstancia, junto a las modificaciones farmacocinéticas y farmacodinámicas consecuencia del envejecimiento, a las enfermedades asociadas (como el deterioro cognitivo o la disminución de la agudeza visual) y a los a veces complejos regímenes terapéuticos, hace a este grupo de población más susceptible a la presentación de reacciones adversas a medicamentos (RAM). En una investigación en urgencias de más de 16.000 pacientes se objetivaron un $3 \%$ de RAM ${ }^{11}$. Además se ha descrito que en la población anciana son las responsables del $10 \%$ de la visitas 
a urgencias $^{12}$. Tener una RAM aumenta la probabilidad de ingreso, (principalmente si es anciano con demencia y dependiente), la estancia media de la hospitalización y el riesgo de muerte ${ }^{11}$.

Por tanto, cuando un paciente anciano visita un SUH y, más aún cuando se le etiqueta de frágil o geriátrico, se le debe considerar un paciente de riesgo. Esto es debido a que, cuando se les compara con los jóvenes, se ha visto que presenta un mayor número de complicaciones, un mayor porcentaje de ingreso, refrecuentación e institucionalización o muerte tras el alta ${ }^{13-17}$.

\section{VALORACIÓN DEL PACIENTE GERIÁTRICO}

El modelo de atención del paciente anciano en los servicios de urgencias no está adaptado al paciente geriátrico ${ }^{18}$. La valoración médica urgente es a menudo breve y dirigida por el motivo de consulta. Por lo tanto, es generalmente unidimensional, centrada en el episodio médico sin reconocer las peculiaridades del anciano y menospreciando la valoración funcional, mental o social. Además, la formación geriátrica del personal de urgencias es limitada ${ }^{19}$.
El paciente geriátrico es un sujeto en el que la valoración médica es más compleja dada la mayor frecuencia de presentación atípica de enfermedades, de comorbilidad y de polifarmacia asociada. Tanto es así que problemas como el infarto, la sepsis o el abdomen quirúrgico en ocasiones son difíciles de identificar ${ }^{20}$. Además, es frecuente no encontrar un único diagnóstico sino que habitualmente encontraremos diferentes procesos clínicos independientes. Todo ello se traduce en la necesidad de evaluaciones médicas más prolongadas, con un mayor número de pruebas complementarias y consultas a otros especialistas, lo que contribuye a aumentar la estancia media en urgencias ${ }^{5}$ y el número de ingresos ${ }^{6}$. De hecho, no es infrecuente el ingreso en salas de observación de los ancianos con quejas no específicas ${ }^{21}$.

\section{Valoración geriátrica integral}

La valoración geriátrica integral (VGI) es una evaluación llevada a cabo por un equipo interdisciplinar que tiene la finalidad de identificar todos los problemas y establecer un plan de cuidados para mejorar la funcionalidad y la calidad de vida del paciente geriátrico (Fig. 1).

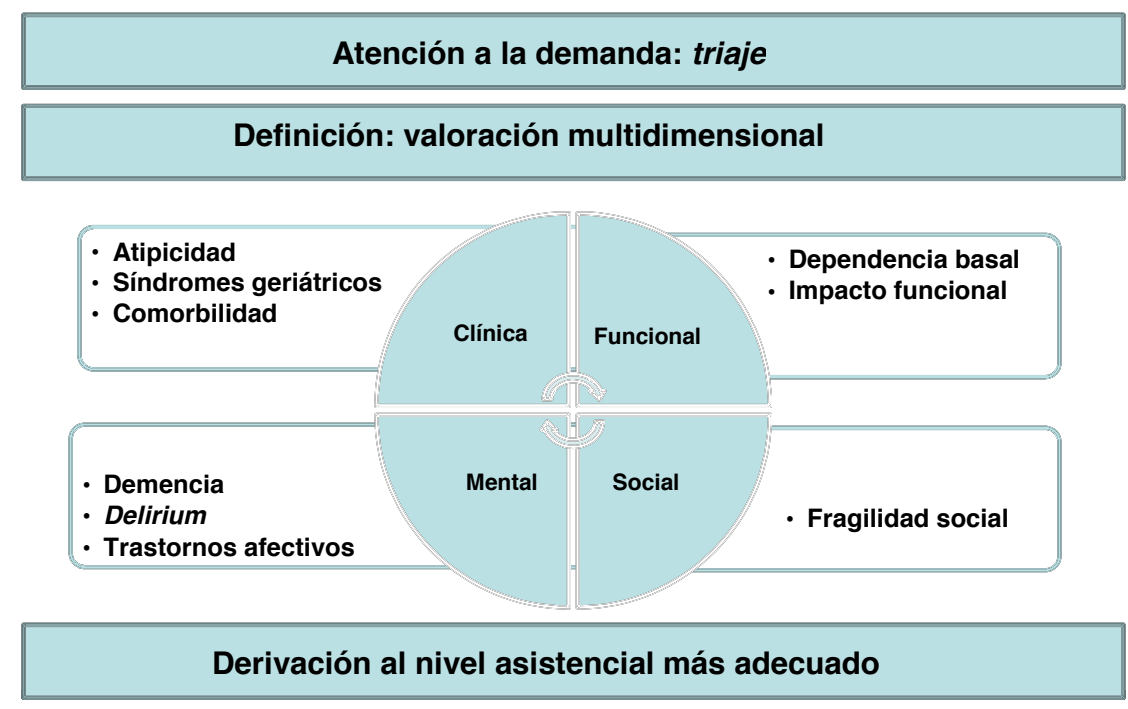

Figura 1. Modelo de atención del paciente anciano en urgencias. 
No existen evidencias hoy que demuestren la efectividad de un modelo concreto de valoración geriátrica aplicado en los servicios de urgencias $^{13}$ e incluso algunos autores creen que no sería el nivel asistencial más adecuado para la VGI. Desde nuestro punto de vista, una VGI adaptada a la dinámica de los SUH nos ayudaría a establecer un plan de cuidados específico a cada paciente anciano con patología médica aguda en urgencias. Esto cobra aún mayor importancia de cara a la realización de maniobras diagnósticas y terapéuticas agresivas, como la reanimación cardiopulmonar, donde la edad no es un factor determinante en la supervivencia sino que lo son otros aspectos como la comorbilidad y la funcionalidad ${ }^{22}$. O, por otro lado, a evitar actitudes nihilistas que conduzcan a la infrautilización de técnicas de eficacia probada por el mero hecho de la edad.

\section{Valoración médica}

Como hemos comentado previamente, la valoración clínica del paciente geriátrico es compleja. La atipicidad de la presentación clínica no sólo se refiere a modificaciones de los síntomas o signos habituales sino también a otras formas de presentación que incluyen cambios en la esfera mental o funcional.

La anamnesis en ocasiones es difícil ya que el paciente es incapaz de suministrar la información necesaria, bien por su estado crítico o por problemas para transmitir una historia coherente. En estos casos, es fundamental la información de un cuidador fiable, del médico de atención primaria o residencia, de la atención extrahospitalaria o de los historiales médicos ${ }^{23}$. En lo referente a la exploración física, no difiere de la realizada en el adulto, pero requiere un mayor minuciosidad, nivel de destreza y observación del paciente.

Es muy importante evaluar la comorbilidad previa. La comorbilidad se entiende como el conjunto de enfermedades y discapacidades existentes previamente y/o que acontecen en el momento de acudir al SUH. Es recomendable apoyarse en es- calas, como la de Charlson, validadas en el ámbito de urgencias ${ }^{24}$.

En lo que respecta a la polifarmacia, recordar que ésta favorece las RAM. Las RAM son en ocasiones difícilmente reconocibles $\mathrm{y}$, en otras, no se interpretan como tales sino a un proceso clínico nuevo, para el cual añadimos un nuevo fármaco y así sucesivamente (cascada de la prescripción). Los fármacos más frecuentemente implicados son los antiinflamatorios no esteroideos, antibióticos, anticoagulantes, diuréticos, betabloqueantes, antagonistas del calcio y los agentes quimioterápicos ${ }^{12}$. Además es importante recordar que existen una serie de fármacos que no son recomendables por criterios de eficacia o seguridad en el paciente anciano (criterios de Beers) ${ }^{25}$.

\section{Valoración funcional}

La dependencia funcional es, en ocasiones, un factor pronóstico más potente que la propia enfermedad. El deterioro funcional agudo detectado en los SUH se relaciona con la reutilización de los propios recursos y con la necesidad de ingreso hospitalario. A su vez, el documentado en unidades de agudos al ingreso se asocia con la mortalidad, la discapacidad y la necesidad de institucionalización ${ }^{26}$.

La valoración funcional en urgencias se puede llevar a cabo mediante escalas que valoran las actividades de la vida diaria (AVD). Para cuantificar las actividades básicas de la vida diaria (ABVD) puede emplearse el índice de Katz o el índice de Barthel y para las actividades instrumentales de la vida diaria (AIVD) el índice de Lawton.

\section{Valoración mental}

Se ha descrito la presencia de deterioro cognitivo en el $25 \%$ de los casos de los ancianos que acuden a urgencias, pero sólo es objetivado en uno de cada cuatro pacientes $^{27}$. El deterioro cognitivo en un marcador de alto riesgo, ya que se asocia a mayor probabilidad de refrecuentación e ingreso hospitalario y de deterioro funcional. Además es un factor de mal pronósti- 
co, asociándose a mayor mortalidad tanto a corto como a largo plazo y más aún en aquellos pacientes donde está presente y no ha sido identificado ${ }^{28}$.

Ante la presencia de un paciente anciano con deterioro cognitivo en urgencias, lo primero que hay que hacer es documentar si existía o no diagnóstico previo de demencia y el tipo de la misma o de otro antecedente psiquiátrico conocido. Posteriormente, tras descartar un estado de coma, siempre habrá que estudiar si el paciente sufre o no un cuadro de delirium como primera posibilidad. En aquellos pacientes con diagnóstico establecido de demencia previo, el diagnóstico diferencial principal del delirium es con la demencia y, más frecuentemente, con los síntomas psicológicos y conductuales asociados a la demencia. La coexistencia de delirium y demencia es frecuente, y no siempre resulta sencillo establecer en qué medida participa cada uno de los elementos en la clínica del paciente en un momento determinado.

En la actualidad no existe evidencia para realizar recomendaciones universalizadas sobre los instrumentos más adecuados para la detección del mismo en urgencias. Respecto al tipo de escala a utilizar debe ser breve, sencilla y adecuada a las circunstancias específicas de cada paciente. Por ejemplo, en referencia a los test cognitivos se puede recurrir a la versión abreviada del test del informador (TIN), el "Six-Item Screener (SIS)», el «Memory Impairment Screen (MIS)", el "1 minute Screen (1MS)" y el Mini-Cog (3-items recall and clock drawing task). Otros que también han demostrado su utilidad en la valoración del cuadro confusional agudo son el "Quick Confusion Scale (QCS)" o el "Confusion Assessment Method (CAM)". Y en la evaluación del nivel de conciencia del paciente en coma el AVPU (alert, responsive to verbal stimuli, to painful stimuli or unresponsive) o la "Glasgow Coma Scale» (GCS) ${ }^{28}$.

\section{Valoración social}

Los pacientes ancianos ingresados por razones sociales tienen mayor mortalidad que los controles de la misma edad y sexo ${ }^{29}$. Además, es muy importante conocer la situación social del paciente anciano de cara a establecer un plan de cuidados. Un buen ambiente y apoyo familiar que acepten la responsabilidad de seguimiento, conjuntamente con la coordinación con atención primaria y los servicios de asistencia domiciliaria, aseguran una disminución de la frecuentación a urgencias y de la pérdida de la funcionalidad. Se considera anciano de alto riesgo social a aquél que vive solo o sin cuidador principal, sin domicilio fijo o que presenta problemas económicos.

Otro problema que hay que tener en cuenta son los abusos y los malos tratos. Identificar al anciano maltratado no es siempre tarea fácil y requiere por parte del profesional un alto índice de sospecha y de búsqueda. Los factores de riesgo son la demencia, un cuidador con alteraciones psicológicas, historia previa de violencia familiar y el ambiente familiar perturbado por causas externas. Ante sospecha o detección de maltrato, habrá que ponerse en contacto con los servicios sociales así como con aquellas otras autoridades implicadas, para una prevención e intervención precoz y eficaz. Tras la exploración y valoración, es necesario si procede hacer un parte de lesiones y remitir al juez, manteniendo informado en todo momento al paciente.

\section{Síndromes geriátricos}

En los ancianos frágiles y pacientes geriátricos es común encontrar los denominados «síndromes geriátricos» $(\mathrm{SG})$. Son un conjunto de cuadros originados por la conjunción de una serie de enfermedades de alta prevalencia en los ancianos y que son frecuente origen de incapacidad funcional o social ${ }^{30}$.

Se han descrito numerosos SG recordados fácilmente como las "íes" de la geriatría. Los más importantes son el delirium y la demencia (intelectual impairment), la inmovilidad (inmobility), las caídas y la inestabilidad (Instability) y la incontinencia urinaria y/o fecal (incontinence) ${ }^{30}$ (Tabla 3). 
Tabla 3. Los síndromes geriátricos.

\begin{tabular}{|l|l|}
\hline Immobility & Inmovilidad \\
Instability & Inestabilidad y caídas \\
Incontinence & Incontinencia urinaria y fecal \\
Intellectual impairment & Deterioro cognitivo \\
Infection & Infecciones \\
Inanition & Desnutrición \\
Impairment of vision and hearing & Alteraciones en vista y oído \\
Impactation & Estreñimiento \\
Isolation (depression)/Insomnio & Depresión/Insomnio \\
Iatrogenesis & Yatrogenia \\
Impotence & Impotencia \\
Inmune deficiency & Inmunodepresión \\
\hline
\end{tabular}

\section{Escalas de detección del paciente anciano de alto riesgo en urgencias}

La detección precoz del paciente anciano de alto riesgo es fundamental para evitar nuevos ingresos, visitas en urgencias y mejorar el nivel de función física y cognitiva. Se han descrito una multitud de instrumentos para el cribado de aspectos funcionales, mentales y sociales en el paciente anciano. Dichas valoraciones no han demostrado clara utilidad en el ámbito urgencias. Por ello, se están desarrollando escalas que ayuden al urgenciólogo a detectar al paciente anciano de alto riesgo.

Se han publicado dos escalas principales de cribado, la ISAR (Identification Senior at Risk) ${ }^{31}$ y la TRST (Triage Risk Screening Tool) ${ }^{32}$. La ISAR recoge aspectos referentes a la dependencia funcional (premórbida y cambio agudo), la polimedicación, el déficit de memoria y visión y la hospitalización reciente. Se considera paciente anciano de riesgo aquél con puntuación mayor o igual a 2. A su vez, la escala TRST incluye la presencia de deterioro cognitivo, vivir solo o no, tener un médico dispuesto o preparado para prestar asistencia, la dificultad para caminar o para las transferencias, la visita a urgencias en los 30 días previos u hospitalización en los últimos 90 días y tener cinco o más medicamentos prescritos. Posteriormente se añadió un nuevo ítem sobre "recomendación profesional» basado en el juicio de la enfermera que aplica el test sobre preocupaciones futuras como posibilidad de incumplimiento o de abuso de sustancias. Se considera paciente en riesgo aquél que presenta deterioro cognitivo o dos o más de los otros cinco factores de riesgo. La ISAR muestra un buen valor predictivo para identificar el anciano con alto riesgo de ingreso, refrecuentación a urgencias, institucionalización y muerte a los 6 meses de la visita a urgencias. La TRST tiene resultados comparables para predecir el riesgo de ingreso, refrecuentación e institucionalización tras el alta ${ }^{29}$.

La detección del paciente anciano de alto riesgo obliga a realizar una valoración geriátrica integral para establecer un plan de cuidados y así poder mejorar los resultados ${ }^{29}$. Como comentamos con anterioridad, hoy en día falta información en la literatura sobre el modelo concreto más efectivo para llevar a cabo dicha valoración en urgencias ${ }^{13}$. Se han descrito varias modelos como la urgencia específica de geriatría, la creación de áreas específicas de atención geriátrica dentro de urgencias, la actuación especializada mediante interconsulta a equipos de soporte geriátrico durante la hospitalización o las consultas de forma ambulatoria. Las áreas de observación (AO) y las unidades de corta estancia de urgencia (UCEU) han demostrado ser alternativas a la hospitalización ya que son unidades diagnósticas y terapéutica de alta resolución y evitan ingresos innecesarios ${ }^{33}$. Según nuestro punto de vista, y en consonancia con otros autores, dichos lugares pueden ser los idóneos para la VGI en aquellos pacientes ancianos de alto riesgo de cara a reducir el número de ingresos y optimizar los cuidados ambulatorios $^{34}$. 


\section{RECOMENDACIONES PRÁCTICAS RELATIVAS AL PACIENTE GERIÁTRICO EN URGENCIAS}

- La valoración del paciente geriátrico en urgencias es compleja, por ello es necesario profundizar en la formación en geriatría de los profesionales sanitarios que atienden al paciente anciano en los SUH.

- El paciente geriátrico es un paciente de alto riesgo. El modelo de atención del paciente geriátrico en urgencias debe ser global y no debe abarcar sólo el episodio médico, sino también los aspectos de la esfera funcional, mental y social.

- Es vital detectar al paciente anciano de alto riesgo o con resultados pobres tras el alta. Para ello puede ser de utilidad escalas de cribado con el fin de seleccionar a aquellos pacientes que se beneficien de una valoración geriátrica integral y así poder diseñar un plan de cuidados específico y dar altas de forma efectiva y segura desde urgencias.

- Es importante desarrollar protocolos de actuación específicos para los diferentes problemas del anciano en el ámbito de urgencias. Por su prevalencia, se deben destacar los síndromes geriátricos, entre ellos el estreñimiento, el cuadro confusional, las caídas o el deterioro funcional así como otras patologías médicas, como la cardiopatía isquémica, la reagudización de problemas crónicos, el ictus, la fractura de cadera o el abuso y el maltrato.

- Las maniobras diagnósticas y terapéuticas no deben estar condicionadas exclusivamente por la edad. Es muy importante considerar otros aspectos como la comorbilidad y la funcionalidad. Además es importante recordar las directrices avanzadas y la futilidad de los tratamientos.

- Para conseguir una intervención exitosa en el paciente geriátrico, es fundamental contar con una exquisita coordinación entre el urgenciólogo, los equipos de valoración geriátrica y los médicos de atención primaria o de las residencias y por supuesto, en caso que sea posible, con la colaboración de la familia o el cuidador principal.

- No se puede recomendar actualmente un modelo funcional o estructural universalizado en la atención del paciente geriátrico en urgencias. Las unidades vinculadas a los $\mathrm{SUH}$, como las salas de observación y las unidades de corta estancia, apuntan como áreas específicas que podrían ser efectivas en el tratamiento de la agudización de procesos crónicos y donde realizar la VGI de cara a reducir el número de ingresos innecesarios y optimizar los cuidados ambulatorios.

- Los servicios de urgencias deberían estar diseñados para el paciente geriátrico. Tendrían que ser una estructura que facilite una estancia confortable y segura que minimizase el riesgo de caídas, con medidas que previniesen las úlceras por presión y con personal sanitario formado específicamente para la atención integral de las personas mayores.

- Es de suma importancia en los próximos años invertir y potenciar la investigación clínica específica de la población anciana, especialmente en el ámbito de los servicios de urgencias.

\section{BIBLIOGRAFÍA}

1. SÁnchez M, Salgado E, Miró O. Mecanismos organizativos de adaptación y supervivencia de los servicios de urgencia. Emergencias 2008; 20: 48-53.

2. Roberts DC, McKay MP, SHAFFer A. Increasing rates of emergency department visits for Elderly Patients in the United States, 1993 to 2003. Ann Emerg Med 2008; 51: 769-774.

3. Xu KT, Nelson BK, Berk S. The changing profile of patients who used Emergency Deparment Services in the United States: 1996 to 2005. Ann Emerg Med. (En prensa). 
4. MoYA MIR MS. Epidemiología de las urgencias del anciano. Monografías Emergencias 2008; 2: 6-8.

5. Llorente S, Arcos PJ, Alonso M. Factores que influyen en la demora del enfermo en un servicio de urgencias hospitalarias. Emergencias 2000; 12: 164-171.

6. Strange GR, Chen EH. Use of emergency departments by elder patients: a five-year follow up study. Acad Emerg Med 1998; 5: 11571162.

7. Fried LP, Tangen CM, Walston J, Newman AB, Hirsch C, Gottdiener J et al. Frailty in older adults: evidence for a phenotype. J Gerontol A Biol Sci Med Sci 2001; 56: M146-156.

8. BORTz WM. A conceptual framework of frailty: a review. J Gerontol A Biol Sci Med Sci 2002; 57: 283-288.

9. Martín Sánchez FJ, González Del Castillo J, ElVIRA C et al. Diferencias del sistema de triaje Manchester entre ancianos y adultos. XXI Congreso Nacional Sociedad Española de Urgencias y Emergencias. Emergencias 2009: 264 (volumen extraordinario).

10. KAHN JH, Magauran B. Trends in geriatric emergency medicine. Emerg Med Clin N Am 2006; 24: 243-260.

11. Zanocchi M, Tibaldi V, Amati D, Francisetti F, Martinelli E, Gonella M et al. Adverse drug reactions as cause of visit to the emergency department: incidence, features and outcomes. Recenti Prog Med 2006; 97: 381-388.

12. Hohl CM, Dankoff J, Colacone A, Afilalo M. Polypharmacy, adverse drug-related events, and potential adverse drug interactions in elderly patients presenting to an emergency department. Ann Emerg Med 2001; 38: 666671.

13. Duaso E, López-Soto AL. Valoración del paciente frágil en urgencias. Emergencias 2009; 21: 362-369.

14. Singal BM, Hedges JR, Rousseau EW, Sanders AB, Berstein E, Mcnamara RM et al. Geriatric patient emergency visits. Part I: comparison of visits by geriatric and younger patients. Ann Emerg Med 1992; 21: 802-807.

15. Strange GR, Chen EH. Use of emergency departments by elder patients: a five-year follow up study. Acad Emerg Med 1998; 5: 11571162.

16. Friedmann PD, Jin L, Karrison TG, Cox Hayley D, MulLiken R, WALter J et al. Early revisit, hospitalization, or death among older persons discharged from the ED. Am J Emerg Med 2001; 19: 125-129.
17. Aminzadeh F, Dalziel WB. Older adults in the emergency department: A systematic review of pattern of use, adverse outcomes, and effectiveness of interventions. Ann Emerg Med 2002; 39: 238-247.

18. Ula Hwang, MD, MPH; R. Sean Morrison, MD. The geriatric emergency department. J Am Geriatr Soc 2007; 55: 1873-1876.

19. MoYa MiR. La formación geriátrica en urgencias. Emergencias 2006; 18: 1-4.

20. REDín JM. Valoración geriátrica integral: evaluación del paciente geriátrico y concepto de Fragilidad. An Sist Sanit Navar 1999; 22 (Supl. 1): 41-50.

21. Ross MA, Compton S, Richardson D, Jones R, NitTIS T, WILSON A. The use and effectiveness of an emergency department observation unit for elderly patients. Ann Emerg Med 2003; 412: 668-677.

22. NARANG AT, SiKkA R. Resuscitation of the elderly. Emerg Med Clin N Am 2006; 24: 261272 .

23. Terrel KM, Brizendine EJ, Bean WF, Giles BK, DAVIDSON JR, Evers $S$ et al. An extended care facility-to-emergency department form improves communication. Acad Emerg Med 2005; 12: 114-118.

24. Olsson T, Terent A, Lind L. Charlson comorbidity index can add prognostic information to rapid emergency medicine score as a predictor of long-term mortality. Eur J Emerg Med $2005 ; 12$ : 220-224.

25. Beers MH, Storrie M, Lee G. Potential adverse drug interaction in the emergency room: an issue of quality of care. Ann Intern Med 1990; 112: 61-64.

26. Fernández Alonso C, Martín Sánchez FJ, Fuentes Ferrer M, González del Castillo J, Verdejo Bravo C, GIL Gregorio P et al. Valor pronóstico de la valoración funcional al ingreso en una unidad de corta estancia de urgencias. Rev Esp Geriatr Gerontol 2010; 45: 63-66.

27. Hustey FM, Meldon SW. The prevalence and documentation of impaired mental status in elderly emergency department patients. Ann Emerg Med 2002; 39: 248-53.

28. Fernández Alonso C, Martín-SÁnchez FJ. Urgencias. En: Gil P, editor. Tratado de neuropsiquiatría. (En prensa).

29. KilshaW L. Australian and new zealand society for geriatric medicine position statement no. 14 guidelines for the management of older persons presenting to emergency departments 2008. Australas J Ageing 2009; 28: 153-157. 
30. GonZÁlez García P. Síndromes geriátricos: conceptos e identificación. Monografías Emergencias 2008; 2: 9-11.

31. McCusker J, Bellavance F, Cardin S, Trepanier S. Screening for geriatric problems in the emergency department: reability and validity. Identification of Seniors at Risk (ISAR) Steering Committee. Acad Emerg Med 1998; 5: 883-893.

32. Meldon SW, Mion LC, Palmer RM, Drew BL, Connor JT, LEWICKI LJ et al. A brief-stratification tool to predict repeat emergency department visits and hospitalizations in older patients discharged from the emergency department. Acad Emerg Med 2003; 10: 224-232.

33. González-Armengol JJ, Fernández Alonso C, Martín-SÁnchez FJ, GonzÁlez del Castillo J, LóPEZ-FARRÉ A, Elvira C et al. Actividad de una unidad de corta estancia en urgencias de un hospital terciario: cuatro años de experiencia. Emergencias 2009; 21: 87-94.

34. Pareja T, Hornillos M, Rodríguez M, Martínez J, Madrigal M, MaUleón C et al. Medical short stay unit for geriatric patients in the emergency department: clinical and healthcare benefits. Rev Esp Geriatr Gerontol 2009; 44: 175-179. 